\title{
Association of Neutrophil-to- Lymphocyte Ratio (NLR) and Platelet-to-Lymphocyte Ratio (PLR) to Disease Activity in Filipino Lupus Patients
}

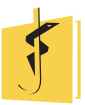 \\ Francis Martin T. Cuenco, MD, \\ Sandra V. Navarra, MD
}

\begin{abstract}
Background: Systemic lupus erythematosus (SLE) is a chronic, multisystem, autoimmune disease characterized by autoantibody production, immune complex deposition and excessive pro-inflammatory cytokine production due to an aberrant and dysfunctional immune system. Disease activity markers for SLE are helpful in the management and prognostication of the disease. The neutrophil-to-lymphocyte ratio (NLR) and platelet-to-lymphocyte ratio (PLR) have been studied as a novel inflammatory marker and prognostic markers for cardiovascular diseases, inflammatory disorders and malignancies.
\end{abstract}

Objective: The aim of the study is to investigate the association of NLR and PLR to disease activity of Filipino patients with SLE.

Methods: This is a cross-sectional study done through a retrospective chart review of 135 Filipino SLE patients divided into two groups. Group 1 (SLEDAI-2K score of $<3$ ) had 64 patients who were in low disease activity/remission and group 2 (SLEDAI$2 \mathrm{~K}$ score of $\geq 3$ ) had 71 patients who were in active disease. Clinical characteristics and disease activity parameters (C3, anti-dsDNA, ESR) and NLR and PLR

Francis Martin T. Cuenco

fmtc32@gmail.com

Section of Rheumatology, Department of Medicine, University of Santo Tomas Hospital, Manila, Philippines were compared in the two groups. Correlations of NLR and PLR with established clinical and laboratory disease activity markers of SLE (C3, anti-dsDNA, SLEDAI-2K scores) were analyzed.

Results: The group 2 or those with active disease had significantly higher NLR $(2.947 \pm 1.756$ vs. $1.868 \pm 0.832$, p-value of $<0.001)$ and PLR (205.9 \pm 122.2 vs. $140.2 \pm 53.0$, p-value of $<0.001$ ) levels compared to group 1. NLR and PLR values were also significantly higher in patients with lupus nephritis. NLR was positively correlated with anti-dsD$\mathrm{NA}(\mathrm{r}=+0.490$, p-value of $<0.001)$ and SLEDAI-2K scores $(r=+0.496$, $p$-value of $<0.001)$. NLR was negatively correlated with $C 3 / r=-0.336$, p-value of $<0.001)$. PLR was also positively correlated with anti-dsDNA $(r=+0.301$, $p$-value of $<0.001)$ and SLEDAI-2K scores $(r=+0.369$, $p$-value $<0.001)$. PLR was also negatively correlated with $C 3$ levels $(r=$ -0.215 , p-value 0.012). Using the ROC curve analysis, the cut-off values in predicting active disease in SLE were 1.968 (sensitivity $77.5 \%$, specificity 75\%) for NLR and 144.53 (sensitivity 63.4\%, specificity $60 \%$ ) for PLR. The cut-off values in predicting lupus nephritis were 2.121 (sensitivity $73.1 \%$, specificity $60 \%$ ) for NLR and 167.0 (sensitivity $65.4 \%$, specificity 68\%) for PLR.

Conclusions: NLR and PLR were significantly higher among Filipino SLE patients with active disease including lupus nephritis reflecting active inflammation. NLR and PLR correlated well with established 
disease activity markers for SLE namely C3, anti-dsDNA, and SLEDAI-2K scores. NLR and PLR could be a useful and convenient disease activity marker for SLE patients.

\section{INTRODUCTION}

Systemic lupus erythematosus (SLE) is the prototypic multisystem autoimmune disease with heterogeneous manifestations and a varied course. $[1,2,3]$ Its exact etiology is unknown and characterized by autoantibody production, immune complex formation and deposition, and excessive pro-inflammatory cytokine production that can affect any organ system of the body. $[1,4,5]$ Inflammation plays a key role in SLE and chronic inflammation is an important pathological process that leads to irreversible damage of the affected organ if left untreated.[4,5] One of the most common and serious complications of SLE is lupus nephritis.[3] Lupus nephritis is the inflammation of the kidneys that is caused by immune complex formation and deposition by SLE. If left untreated, lupus nephritis can progress to end-stage renal disease and caused significant mortality and morbidity. [3]

Many laboratory tests have been used as indicators of disease activity in SLE and as a guide for clinicians in the management of SLE. These laboratory tests include increased anti-dsDNA, low complement levels (C3 and C4), and increased inflammatory markers such as erythrocyte sedimentation rate (ESR). Many tools have also been validated to measure disease activity in SLE. $[11,12]$. SLE Disease Activity Index - 2000 (SLEDAI-2K) is a validated tool used to measure disease activity in SLE. $[11,12]$ It measures the global disease activity in SLE and predictor of mortality. The higher the SLEDAI-2K score, the higher is the predictive mortality of the patient. $[11,12]$ It is one of the most commonly used global disease activity measures in longitudinal observational studies and clinical trials. It is a modification of the original SLEDAl consisting of 24 of the most important disease descriptors and has been a guide for clinicians in the management of their SLE patients and researchers doing clinical trials. $[11,12]$ Targets for the management of SLE patients have also used SLEDAI-2K, particularly remission or low disease activity. Remissions have been described as SLEDAI-2K score of 0 while low disease activity state has been described as SLEDAI-2K score of $<3$. $[11,12]$
NLR and PLR have been studied as a novel inflammatory marker and prognostic markers for cardiovascular diseases, inflammatory disorders, and malignancies.[4-10] NLR is calculated as the absolute count of neutrophils divided by the absolute count of lymphocytes. $[4,5,7]$ PLR is calculated as the absolute platelet count divided by the absolute lymphocyte count. $[4,5,7]$ NLR and PLR have also been recently studied in association with autoimmune diseases namely psoriasis, rheumatoid arthritis, and SLE. $[4,5,10]$ NLR and PLR can be calculated easily, conveniently, and inexpensively in comparison with other disease markers of SLE. New studies regarding the correlation of NLR and PLR to SLE have also been done abroad. In a study by Wu, et al., NLR and PLR showed a positive correlation with SLE disease activity. [4] In a study by Li, et al., NLR has been shown to be independently associated with SLE and lupus nephritis. [5]

The aim of this study is to determine the association and correlation of NLR and PLR to disease activity in Filipino SLE patients and in the future can help clinicians, especially those that are in the provinces wherein more sophisticated disease markers for SLE are not readily available. In the investigator's knowledge, this is the first local study correlating NLR and PLR to SLE disease activity.

\section{MATERIALS AND METHODS}

This is a cross-sectional study done through a retrospective chart review through the nonprobability sampling of SLE patients aged 19 years and older who were seen at the UST Hospital from January 2013 to December 2018. The patients included in the study satisfied either the 1997 ACR criteria or the 2012 Systemic Lupus Collaborating Clinics (SLICC) classification criteria for SLE. The disease activity was assessed for each group using the SLEDAI$2 \mathrm{~K}$ system. Those who satisfied the inclusion criteria for the study were divided into two groups - those who are in remission or low disease activity (SLEDAI$2 \mathrm{~K}$ score of less than 3) served as the control group and those who have clinical active disease (SLEDAI$2 \mathrm{~K}$ score of more than or equal to 3 ). The baseline demographics, clinical characteristics and laboratory results were recorded and compared for each group. NLR and PLR were computed for each group and its association and correlation with disease activity were determined. Other laboratory tests for 
disease activity in SLE (C3, anti-dsDNA, ESR) and presence of active lupus nephritis were also correlated to NLR and PLR.

\section{Inclusion Criteria}

The subjects of this study consisted of Filipino patients aged 19 or above who were diagnosed with SLE based on either the 1997 ACR revised criteria for diagnosis or the 2012 SLICC classification criteria for SLE who were seen at the UST Hospital from January 2013 to December 2018.

\section{Exclusion Criteria}

The exclusion criteria set for the study included patients who had overlapping autoimmune diseases including scleroderma, Sjogren's syndrome, rheumatoid arthritis (RA), mixed connective tissue disease (MCTD), idiopathic inflammatory myopathy, as well as infectious processes mimicking lupus. Patients with hematologic disease or those who had received blood transfusion in the past 6 months, malignant diseases and those using medical treatment affecting white blood cell (WBC) count were also excluded in the study. Patients with acute infection or other concomitant inflammatory diseases that may affect the NLR and PLR were also excluded in the study.

\section{Statistical Analysis}

All statistical analyses were performed using the SPSS statistical software (version 21.0, IBM SPSS, Chicago, IL, USA). Descriptive statistics for continuous variables were expressed in mean and standard deviation; categorical variables were expressed in frequency and percentages. Student t-test or MannWhitney U-test was used to compare between two groups according to the distribution state. The Chisquare test was used to compare proportions in different groups. The Spearman correlation coefficient was computed to examine the association between two continuous variables. Receiver-Operating Characteristics (ROC) curves were also used to determine the sensitivity and specificity of NLR and PLR in predicting active SLE and lupus nephritis. Statistical significance was defined as a $\mathrm{P}$ value less than 0.05 .

\section{Ethical Consideration}

The research was conducted upon the approval of the University of Santo Tomas Institutional Review Board in accordance with the ethical principles set out in the Declaration of Helsinki 2015.

\section{RESULTS}

\section{Demographic Characteristics}

A total of 135 Filipino SLE patients were included in the study. They were assigned into two groups namely group 1 (those on remission or low disease activity, SLEDAI-2K score $<3$ ) and group 2 (those with active disease, SLEDAI-2K score $\geq 3$ ). A total of 64 patients (63 females, 98.4\%) were included in group 1 with a mean age of $34.42 \pm 10.36$ years, range 19-58. The mean age of SLE diagnosis was $24.98 \pm 9.13$ years and the mean duration of SLE was $9.44 \pm 6.08$ years. For group 2 , there were a total of 71 patients assigned (64 females, $90.1 \%$ ) with a mean age of $35.14 \pm 9.04$ years, range 20 59. The mean age of SLE diagnosis for group 2 was $26.18 \pm 7.89$ years and the mean duration of SLE was $8.69 \pm 5.48$ years. There were no significant differences in the demographic characteristics of the two groups in the study.

\section{NLR and PLR Levels and SLE Disease Activity}

Based on the clinical characteristics of the two groups, group 2 or those with active SLE have higher

Table 1. SLE Patients Demographic Characteristics

\begin{tabular}{|c|c|c|c|}
\hline & $\begin{array}{c}\text { Group } 1 \text { (SLEDAI-2K <3) } \\
n=64\end{array}$ & $\begin{array}{c}\text { Group } 2 \text { (SLEDAI-2K } \geq 3 \text { ) } \\
n=71\end{array}$ & P-value \\
\hline Age (years) & $34.42 \pm 10.36$ & $35.14 \pm 9.04$ & 0.667 \\
\hline Sex & $\begin{array}{c}\text { Female } 63(98.4 \%) \\
\text { Male } 1(1.6 \%)\end{array}$ & $\begin{array}{l}\text { Female } 64(90.1 \%) \\
\text { Male } 7(9.9 \%)\end{array}$ & \\
\hline $\begin{array}{l}\text { Age at diagnosis of SLE } \\
\text { (years) }\end{array}$ & $24.98 \pm 9.13$ & $26.18 \pm 7.89$ & 0.415 \\
\hline SLE duration (years) & $9.44 \pm 6.08$ & $8.69 \pm 5.48$ & 0.454 \\
\hline
\end{tabular}


Table 2. Clinical and laboratory characteristics of SLE patients including NLR and PLR

\begin{tabular}{|c|c|c|c|}
\hline & $\begin{array}{c}\text { Group } 1 \text { (SLEDAI-2K <3) } \\
n=64\end{array}$ & $\begin{array}{c}\text { Group } 2 \text { (SLEDAI-2K } \geq 3 \text { ) } \\
n=71\end{array}$ & P-value \\
\hline SLEDAI-2K score & $1.16 \pm 0.996$ & $8.10 \pm 4.861$ & $<0.001$ \\
\hline C3 (g/L) & $1.00 \pm 0.14$ & $0.736 \pm 0.20$ & $<0.001$ \\
\hline Anti-dsDNA (IU/mL) & $191.1 \pm 178.9$ & $678.49 \pm 315.8$ & $<0.001$ \\
\hline Active lupus nephritis & $0(0 \%)$ & $26(36.6 \%)$ & \\
\hline $\mathrm{ESR}(\mathrm{mm} / \mathrm{hr})$ & $31.09 \pm 11.2$ & $43.5 \pm 22.8$ & 0.091 \\
\hline WBC (x 109/L) & $6.037 \pm 1.59$ & $6.43 \pm 2.29$ & 0.003 \\
\hline Neutrophil (x 109/L) & $3.53 \pm 1.09$ & $4.28 \pm 1.69$ & 0.002 \\
\hline Lymphocyte (x 109/L) & $2.04 \pm 0.67$ & $1.67 \pm 0.73$ & 0.401 \\
\hline Platelets (x 109/L) & $262.22 \pm 66.8$ & $285.27 \pm 98.5$ & 0.047 \\
\hline NLR & $1.868 \pm 0.832$ & $2.947 \pm 1.756$ & $<0.001$ \\
\hline PLR & $140.2 \pm 53.0$ & $205.9 \pm 122.2$ & $<0.001$ \\
\hline
\end{tabular}

Table 3. NLR and PLR levels and active lupus nephritis

\begin{tabular}{lccc}
\hline & $\begin{array}{c}\text { Group } \mathbf{1} \text { (w/o active nephritis) } \\
\mathbf{n}=\mathbf{1 0 9}\end{array}$ & $\begin{array}{c}\text { Group } \mathbf{2} \text { (with active nephritis) } \\
\mathbf{n}=\mathbf{2 6}\end{array}$ & P-value \\
\hline NLR & $2.20 \pm 1.07$ & $3.41 \pm 2.52$ & $<0.001$ \\
PLR & $165.6 \pm 93.3$ & $213.2 \pm 123.1$ & 0.194 \\
\hline
\end{tabular}

Table 4. Spearman correlation of NLR and PLR to clinical and laboratory characteristics of SLE patients

\begin{tabular}{lcccccc}
\hline $\begin{array}{l}\text { Spearman } \\
\text { Correlation }\end{array}$ & \multicolumn{2}{c}{ SLEDAI-2K } & \multicolumn{2}{c}{ NLR } & \multicolumn{2}{c}{ PLR } \\
\hline & $\mathrm{R}$ & $\mathrm{p}$-value & $\mathrm{r}$ & $\mathrm{p}$-value & $\mathrm{r}$ & $\mathrm{p}$-value \\
C3 (g/L) & -0.634 & $<0.001$ & -0.336 & $<0.001$ & -0.215 & 0.012 \\
Anti-dsDNA & +0.727 & $<0.001$ & +0.490 & $<0.001$ & +0.301 & $<0.001$ \\
(IU/mL) & & & & & & \\
SLEDAl-2K & & & +0.496 & $<0.001$ & +0.369 & $<0.001$ \\
NLR & +0.496 & $<0.001$ & & & +0.608 & $<0.001$ \\
PLR & +0.369 & $<0.001$ & +0.608 & $<0.001$ & & \\
\hline
\end{tabular}

SLEDAI-2K scores and more patients with active lupus nephritis. All the conventional disease activity markers for SLE (C3, anti-dsDNA, and ESR) were observed clearly in group 2. Group 2 have lower C3 levels compared with group 1 (0.736 \pm 0.20 $\mathrm{g} / \mathrm{L}$ vs. $1.00 \pm 0.14 \mathrm{~g} / \mathrm{L})$. Group 2 also had higher anti-dsDNA levels $(678.49 \pm 315.8 \mathrm{lU} / \mathrm{mL}$ vs. $191.1 \pm 178.9 \mathrm{IU} / \mathrm{mL})$ and higher ESR levels $(43.5$ $\pm 22.8 \mathrm{~mm} / \mathrm{hr}$ vs $31.09 \pm 11.2 \mathrm{~mm} / \mathrm{hr}$ ) than group 1. In this study, those with active SLE (group 2) have significantly higher NLR $(2.947 \pm 1.756$ vs $1.868 \pm$ 0.832 , p-value of $<0.001)$ and PLR $(205.9 \pm 122.2$ vs. $140.2 \pm 53.0$, p-value of $<0.001$ ) levels compared to those in low disease activity or remission (group 1).

\section{NLR and PLR Levels and Lupus Nephritis}

In this study, the association of NLR and PLR levels to active lupus nephritis were also evaluated. The patients were also divided into those with active nephritis and those without nephritis. The NLR levels of those with active nephritis were significantly elevated compared to those without nephritis while the PLR levels were also elevated but not statistically significant.

\section{Spearman Correlation of NLR and PLR and Clinical Characteristics of SLE Patients}

NLR was positively correlated with anti-dsDNA $(r=+0.490, p$-value $<0.001)$ and SLEDAI-2K scores 
Table 5. Cut-off values for NLR and PLR using ROC curve analysis for SLE patients with active SLE and lupus nephritis

\begin{tabular}{lccc}
\hline ROC curve for active SLE & $\begin{array}{l}\text { Cut-off } \\
\text { value }\end{array}$ & Sensitivity & Specificity \\
\hline NLR & 1.968 & $77.5 \%$ & $75 \%$ \\
PLR & 144.53 & $63.4 \%$ & $60 \%$ \\
ROC curve for lupus & & & \\
nephritis & & & \\
NLR & 2.121 & $73.1 \%$ & $60 \%$ \\
PLR & 167.0 & $65.4 \%$ & $68 \%$ \\
\hline
\end{tabular}

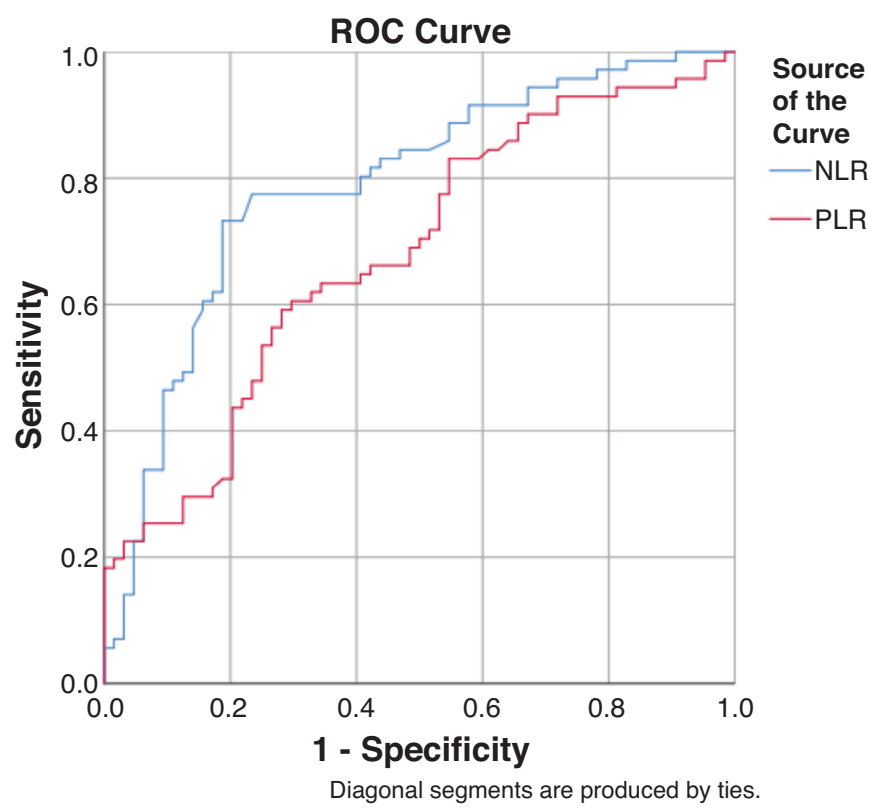

Figure 1. ROC curve for NLR and PLR and active SLE

( $r=+0.496$, p-value $<0.001)$. NLR was negatively correlated with C3 ( $r=-0.336$, $p$-value $<0.001)$. PLR was also positively correlated with anti-dsDNA ( $r=+0.301$, $p$-value $<0.001)$ and SLEDAl-2K scores $(r=+0.369$, p-value $<0.001)$. PLR was also negatively correlated with $C 3$ levels $(r=-0.215$, p-value $0.012)$. All of the correlations of NLR and PLR were statistically significant.

\section{Receiver-Operating Characteristics (ROC) Curves for NLR and PLR}

The ROC curves for NLR and PLR compared to active SLE and lupus nephritis are described in the figures and tables below. The cut-off values in predicting active disease in SLE using the ROC curve analysis at optimal sensitivity and specificity were 1.968 (sensitivity $77.5 \%$, specificity $75 \%$ ) for NLR and 144.53 (sensitivity $63.4 \%$, specificity 60\%) for PLR. The cutoff values in predicting lupus nephritis using the ROC

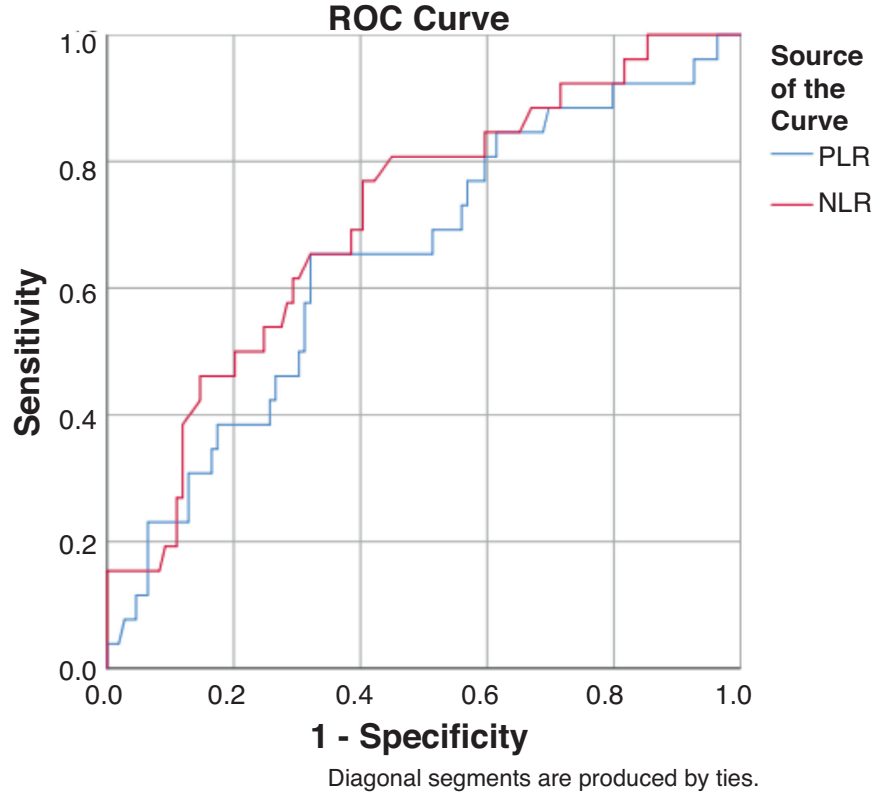

Figure 2. ROC curve for NLR and PLR and lupus nephritis

curve analysis were 2.121 (sensitivity $73.1 \%$, specificity 60\%) for NLR and 167.0 (sensitivity 65.4\%, specificity 68\%) for PLR.

\section{DISCUSSION}

The aim of the study was to investigate the association of NLR and PLR to disease activity of Filipino patients with SLE. It looks at NLR and PLR as a possible novel marker for disease activity in SLE patients. This was done by comparing NLR and PLR with standard and validated clinical and laboratory parameters used by clinicians and researchers in evaluating disease activity in SLE patients. In this study, NLR and PLR were correlated with SLEDAI-2K scores, C3 levels, anti-dsDNA levels and ESR. NLR and PLR were also correlated to active lupus nephritis. This was done through retrospective chart review and the patients included in the study were assigned either with low-disease activity/remission (group 1, SLEDAI-2K score $<3$ ) or with active disease (group 2, SLEDAI$2 \mathrm{~K}$ score $\geq 3$ ). There is no significant difference between the two groups based on demographic characteristics.

Those with active disease (group 2) have higher SLEDAI-2K scores as expected. Anti-dsDNA levels were significantly higher and C3 levels were significantly lower in group 2 . This was expected in the active disease state of SLE wherein there is increased dsDNA binding of autoantibodies and active consumption of complement due to deposi- 
tion of immune complexes. The ESR levels, an acute phase reactant and marker of inflammation, were also higher in group 2 but not statistically significant. NLR and PLR were also significantly elevated in group 2, which reflects the possibility of NLR and PLR as disease activity markers in SLE. The high NLR and PLR values in SLE can be explained by the immune dysregulation, increased cytokines and inflammatory process that happens during active disease. $[4,5]$. WBCs or leukocytes have an important role in the inflammatory process and neutrophils make up the majority of WBCs. [4,5] Neutrophils as part of the innate immune system also secrete different cytokines and inflammatory mediators in response to antigens or tissue injury. Platelets also have a role in the inflammatory process and it also secretes mediators that are active in inflammation. Neutrophils and platelets contribute to the inflammation seen in rheumatic diseases such as SLE. [4,5]

NLR and PLR have been studied as a novel inflammatory marker and prognostic markers for cardiovascular diseases, inflammatory disorders, and malignancies. [4-10]. NLR and PLR can be calculated easily from a complete blood count and has an added advantage of being cheaper and readily available compared to other markers of disease activity in SLE. NLR and PLR also have the advantage of not being affected by age, hemoglobin level, and are relatively stable.[4-7] In the subgroup analysis, the study also compared the NLR and PLR levels of SLE patients with active nephritis to those without nephritis. NLR levels were significantly higher in patients with lupus nephritis compared to those without nephritis. PLR levels were also higher in patients with lupus nephritis but not statistically significant. Lupus nephritis represents an active disease process and it connotes an inflammatory process that would explain the higher NLR and PLR values.[5]

To test for the association of NLR and PLR to disease activity in SLE, Spearman correlation coefficient of NLR and PLR to SLEDAI-2K scores, anti-dsDNA, and $C 3$ levels were computed. Based on the results, NLR and PLR have a positive correlation with SLEDAI-2K scores and anti-dsDNA and negative correlation with C3 levels. All of the correlation coefficients were statistically significant which demonstrated a good correlation with the variables. Both NLR and PLR correlated well with established disease activity markers for SLE. This is also similar to the results of the other studies done abroad.[4-7]

The study also used ROC curve analysis to show which cut-off values at optimal sensitivity and specificity would correlate well or predict active SLE and lupus nephritis. Based on the results, the cutoff values in predicting active disease in SLE were 1.968 (sensitivity $77.5 \%$, specificity $75 \%$ ) for NLR and 144.53 (sensitivity $63.4 \%$, specificity 60\%) for PLR. The cut-off values in predicting lupus nephritis using the ROC curve analysis were 2.121 (sensitivity $73.1 \%$, specificity $60 \%$ ) for NLR and 167.0 (sensitivity 65.4\%, specificity 68\%) for PLR.

The limitations of the study include its retrospective cross-sectional study design. This is also a study done from a single center and measurements of NLR and PLR were based on a single measurement of complete blood count which may not be reflective of the disease state over a period of time. Prospective multi-center studies are recommended in the future for further validation of these results.

\section{CONCLUSION}

This study demonstrated that NLR and PLR were significantly higher among Filipino SLE patients with active disease including lupus nephritis reflecting active inflammation. NLR and PLR correlated well with established disease activity markers for SLE namely C3, anti-dsDNA, and SLEDAI-2K scores. NLR and PLR could be a useful and convenient disease activity marker for SLE patients. 


\section{REFERENCES}

1. Hochberg MC. Updating the American College of Rheumatology revised criteria for the classification of systemic lupus erythematosus. Arthritis Rheum. 1997;40:1725.

2. Petri M, Orbai A-M, Alarcón GS, Gordon C, Merrill JT, Fortin PR, et al. Derivation and validation of systemic lupus international collaborating clinics classification criteria for systemic lupus erythematosus. Arthritis Rheum. 2012;64:2677-86.

3. Hahn BH, McMahon M, Wilkinson A, Wallace WD, Daikh DI, Fitzgerald JD, et al. American College of Rheumatology guidelines for screening, case definition, treatment and management of lupus nephritis. Arthritis Care Res. 2012;64:797-808.

4. Wu Y, Chen Y, Yang X, Chen L, Yang Y. Neutrophil-to-lymphocyte ratio (NLR) and platelet-to-lymphocyte ratio (PLR) were associated with disease activity in patients with systemic lupus erythematosus. International Immunopharmacology. 2016;36:94-9.

5. Li L, Xia Y, Chen C, Cheng P, Peng C. Neutrophil-lymphocyte ratio in systemic lupus erythematosus disease: a retrospective study. Int J Clin Exp Med. 2015;8(7):1 1026-31.

6. Farouk HM, Rahman MA, Mohamed NA, Attia OB. Neutrophil/lymphocyte and platelet/lymphocyte ratios and their relation with disease activity in systemic lupus erythematosus patients. Egyptian Journal of Hospital Medicine. 20170ct 1;69(1):1764-1769.

7. Lee YH, Song GG. Association of neutrophil to lymphocyte ratio, platelet to lymphocyte ratio, and mean platelet volume with systemic lupus erythematosus disease activity: a meta-analysis. Journal of Rheumatic Diseases. 2017 Oct $1 ; 24(5): 279-86$.
8. Tamhane UU, Aneja S, Montgomery D, Rogers EK, Eagle KA, Gurm HS. Association between admission neutrophil to lymphocyte ratio and outcomes in patients with acute coronary syndrome. Am J Cardiol. 2008;102:653-60.

9. Templeton AJ, McNamara MG, Šeruga B, Vera-Badillo FE, Aneja $P$, Ocaña A, et al. Prognostic role of neutrophil-tolymphocyte ratio in solid tumors: a systematic review and meta-analysis. JNCl: Journal of the National Cancer Institute. 2014 Jun $1 ; 106(6)$ :dju124.

10. Mercan R, Bitik B, Tufan A, Bozbulut UB, Atas N, Ozturk $M A$, et al. The association between neutrophil/lymphocyte ratio and disease activity in rheumatoid arthritis and ankylosing spondylitis. J Clin Lab Anal. 2016;30:597-601.

11. Gladman DD, Ibanez D, Urowitz MB: Systemic lupus erythematosus disease activity index 2000. J Rheumatol. 2002;29(2):288-91.

12. Touma Z, Urowitz MB, Gladman DD. SLEDAI-2K for a 30-day window. Lupus. 2010;19(1):49-51.

Open Access This article is licensed under a
Creative Commons Attribution 4.0 International
License, which permits use, sharing, adaptation, distribution and
reproduction in any medium or format, as long as you give ap-
propriate credit to the original author(s) and the source, provide
a link to the Creative Commons license, and indicate if changes
were made. The images or other third party material in this arti-
cle are included in the article's Creative Commons license, un-
less indicated otherwise in a credit line to the material. If materi-
al is not included in the article's Creative Commons license and
your intended use is not permitted by statutory regulation or ex-
ceeds the permitted use, you will need to obtain permission di-
rectly from the copyright holder. To view a copy of this license,
visit http://creativecommons.org/licenses/by/4.0/.

